\title{
MODELAGEM MULTICRITÉRIO PARA A MENSURAÇÃO DO DESEMPENHO DE FORNECEDORES PARA INDÚSTRIAS DE BASE TECNOLÓGICA
}

\author{
Alvaro Luiz Neuenfeldt Júnior \\ Universidade Federal De Santa Maria; Av Roraima 1000, Prédio 07, Sala 300, 97105-900 \\ alvjr2002@hotmail.com \\ Julio Cezar Mairesse Siluk \\ Universidade Federal De Santa Maria; Av Roraima 1000, Prédio 07, Sala 300, 97105-900 \\ jsiluk@ufsm.br \\ Daniel Marques da Silva Chaves \\ Universidade Federal De Santa Maria; Av Roraima 1000, Prédio 07, Sala 300, 97105-900 \\ danielmschaves@gmail.com \\ Marlon Soliman \\ Universidade Federal De Santa Maria; Av Roraima 1000, Prédio 07, Sala 300, 97105-900 \\ marlonsoliman@gmail.com \\ Elpídio Oscar Benitez Nara \\ Universidade de Santa Cruz do Sul; Av Independência 2293, Prédio 53, Sala 5340, 96815-900 \\ elpidio@unisc.br
}

\section{Resumo}

O processo de decisão que envolve a seleção de fornecedores pode ser considerado um aspecto crítico para a criação de vantagens competitivas em empresas de base tecnológica. Assim, este artigo teve por objetivo propor uma modelagem matemática que compreenda a mensuração do desempenho relativo dos fornecedores para indústrias incubadas de base tecnológica, onde a estrutura metodológica foi contemplada, primeiramente, a partir do levantamento de informações contidas na literatura científica, e posteriormente, realizou-se a construção do sistema de mensuração com base nos pressupostos permeados pela Análise Hierarquica de Processos (AHP). A modelagem foi aplicada em três empresas instaladas na Incubadora Tecnológica de Santa Maria (ITSM), situada junto a Universidade de Santa Maria (UFSM), onde constatou-se a ferramenta é capaz de auxiliar as indústrias do ramo tecnológico ao permitir um embasamento maior do nível do desempenho atingido por seus principais fornecedores. Constatou-se, ao final, que a ferramenta proposta por essa pesquisa colabora na busca pelo aumento da eficiência operacional ao propor uma modelagem que sirva de apoio à tomada de decisão gerencial e, consequentemente, das ações e estratégias a serem desenvolvidas pela gestão da empresa no futuro.

Palavras-Chaves: Avaliação de Fornecedores; Empresas de Base Tecnológica; Incubadoras Tecnológicas; Sistema de Mensuração de Desempenho; Analise Hierarquica de Processos.

\section{Abstract}

The decision process that involves the selection of suppliers can be considered a critical aspect for the creation of competitive advantage in technology-based firms. Thus, this article aims to propose a mathematical modeling to measure the relative performance of suppliers for incubated technology-based firms, where the methodological framework was 
contemplated, firstly, from the collection of information contained in the scientific literature, and later, there was the construction of the measurement system based on the assumptions pervaded by hierarchical Analysis Process (AHP). The model was applied in three companies installed in the Technology Incubator of Santa Maria (ITSM), located in the Federal University of Santa Maria (UFSM), where it was found that the tool is able to assist technological industries by allowing greater foundation of level of performance achieved by their main suppliers. We found that, in the end, the tool proposed by this research collaborates in the search for increasing operational efficiency by proposing a model that serves to support management decision making and, therefore, the actions and strategies to be developed by the management company in the future.

Keywords: $\quad$ Supplier Evaluation; Technology-based Firms; Technological Incubators; Performance Measurement System; Analytic Hierarchical Process.

\section{INTRODUÇÃO}

Atualmente as organizações devem estar conscientes quanto aos aspectos relativos ao nível de importância do fator qualidade no momento da aquisição de matérias-primas, sendo esta considerada como um dos principais pontos considerados quando da busca por vantagens competitivas de mercado [17; 31]. Diante desse ponto, a qualidade dos produtos fornecidos pode ser considerada como fundamental para a definição do nível de qualidade do produto final ou serviço oferecido aos clientes $[10 ; 21 ; 36]$.

Para tanto, a mensuração de desempenho está contida ao contexto de forma a colaborar para que haja um maior controle da maneira com que ocorre esse tipo de atividade, alinhado aos critérios considerados como determinantes para se afirmar o sucesso ou fracasso de uma fornecedora, questão essa ainda mais relevante ao se tratar o tema sob a ótica das indústrias de tecnologia $[16 ; 20 ; 28]$. Entretanto, tem-se observado que empresas de base tecnológica (EBTs) neessitam lançar mão de métodos eficazes e simples para avaliar os seus fornecedores, o que possibilita a efetiva gestão dos recursos materiais e humanos para o cumprimento das suas atividades fim $[2 ; 29 ; 30]$.

Por consequência das oportunidades encontradas, o problema que permeia a condução da pesquisa envolve o seguinte questionamento: "Como elaborar uma ferramenta capaz de apoiar a tomada de decisão em relação à escolha de fornecedores em indústrias incubadas e que comercializem produtos caracterizados como de base tecnológica?". A partir do problema abordado, o objetivo central da proposta é propor uma modelagem matemática

que compreenda a mensuração do desempenho relativo dos fornecedores para indústrias incubadas de base tecnológica, contribuindo-se assim para o fortalecimento destas, visto o potencial econômico associado a estas empresas e o papel que exercem na transferência de tecnologia.

\section{METODOLOGIA}

A estrutura metodológica do trabalho está contemplada a partir do cumprimento de quatro etapas sequenciais, conforme mostra a Figura 1, onde primeiramente foram levantadas informações através da bibliografia relacionada a atividade de avaliação de fornecedores, baseado em relatos previamente estabelecidos principalmente nas obras de [3], [4], [8], [13], [18], [25] e [36], com objetivo de identificar percepções e variáveis relativas as influências dos critérios estabelecidos em relação ao ramo de indústrias de base tecnológicas.

Com isso possibilitou-se avançar para a próxima etapa, da modelagem do sistema de medição de desempenho (SMD), a partir da transcrição das informações obtidas em uma estrutura analítica organizada em fatores Cta denominados por Pontos de Vistas Fundamentais $(\mathrm{PVF})(t=1)$, para os localizados no primeiro nível da hierarquia, e 


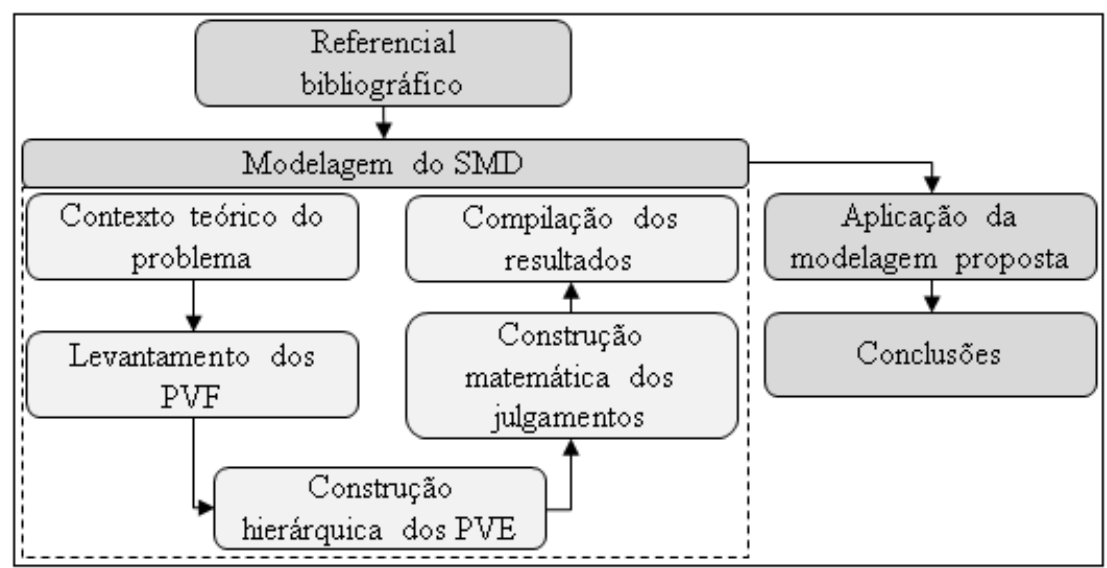

Figura 1 - Etapas metodológicas da pesquisa

Elementares (PVE) $t=\{2,3, \ldots n\}$ aos demais, que tem por finalidade orientar para redução do risco no momento de se mensurar o nível de contribuição dos fornecedores para o processo, de modo que $a$ representa a identidade do elemento em relação ao contexto e $t$ o nível em que o mesmo se encontra. Para tanto, houve a demanda pela utilização de um método capaz de estruturar tal tarefa, foi proposta a abordagem através da do modelo Function Analysis System Technique (FAST) [5; 24].

Com a hierarquia dos pontos de vistas realizada, houve a construção matemática dos julgamentos, a partir do dimensionamento das taxas de substituição para o grau de relevância dos PVFs e PVEs, estruturada em duas partes, através da aplicação de diagnósticos fechados, no qual estão diretamente vinculados aos PVEs extraídos. A diferença entre eles é que o primeiro (Diagnóstico 1) trata da hierarquização dos PVEs $\left(c_{\alpha} 2 a\right)$ e, consequentemente, dos PVFs $\left(c_{\alpha} 1 a\right)$, e deve ser submetido ao gerente da incubadora tecnológica, por este possuir uma maior experiência no que tange a mensuração da forma com que fatores podem afetar ao contexto.

Já o segundo (Diagnóstico 2) visa ser aplicado unicamente aos gestores das empresas envolvidos com a aquisição de insumos, de forma a atribuir pontos aos $f$ fornecedores conforme o seu desempenho em relação a cada um dos PVEs $\left(p f_{C 2 a}\right)$. Com o objetivo de facilitar a compreensão, no momento da aplicação da pesquisa foi adotada uma escala de valores familiar e de fácil compreensão, não negativa, identificada como $\alpha, \alpha=\{1,2, \ldots, 10\}$, conceituada com base nos pressupostos da Escala Likert.

O conjunto destes diagnósticos permite a diagramação de um painel de controle que contempla a relação do nível de desempenho dos forncecedores escolhidos, seja globalmente ou especificamente aos diversos PVFs e PVEs que fazem parte da modelagem. Para tanto, foi utilizada para a estruturação matemática da avaliação os métodos baseados nos pressupostos da AHP, em específico a sua versão clássica, desenvolvida por [32], e uma de suas variantes, a Multiple Attribute Hierarchical Process (MAHP), concebida por [23]. A escolha pela utilização destes métodos se deve a possibilidade de aumentar o nível de confiabilidade do arranjo das decisões tomadas ao decorrer das matrizes de julgamentos, de maneira a tomar por referência os resultados obtidos em ambas as técnicas.

A fim de garantir que a modelagem possa gerar resultados que remetam consideravelmente a realidade em relação aos dados disponibilizados, foi proposta a utilização da razão de consistência dos julgamentos estabelecidos nas análises hierárquicas do problema, com base nos pressupostos descritos por [34], de modo a verificar o nível de confiança quando da aplicação prática da modelagem.

Em decorrência da inserção do pesquisador no contexto da pesquisa e, principalmente, pelo envolvimento do pesquisador e dos membros da organização em torno de um interesse comum, adotou-se para a aplicação prática três empresas $(e=1 ; 2 ; 3)$ instaladas na Incubadora Tecnológica de Santa Maria (ITSM), situada junto a Universidade 
Federal de Santa Maria (UFSM), pois as mesmas já apresentam condições de relatar com mais segurança e confiabilidade as experiências obtidas para opinar acerca de dois dos seus principais fornecedores $(f=1 ; 2)$.

\section{ELABORAÇÃO DA MODELAGEM}

\subsection{CONTEXTO TEÓRICO DO PROBLEMA}

Uma das principais dificuldades nas pesquisas envolvendo empresas de base tecnológica reside justamente na ausência de uma definição padronizada dessas, sendo encontradas diversas abordagens nesse sentido [37]. No entanto, as EBTs podem ser melhor entendidas a partir de características comuns entre as mesmas, tais como operações de pequena escala, desenvolvimento de produtos de elevado conteúdo tecnológico, foco de atuação em nicho, estratégia de diferenciação, e utilização de recursos humanos com elevado grau de escolaridade $[19 ; 22 ; 37]$.

No cenário atual brasileiro as empresas de base tecnólogica se destacam por sua capacidade inovativa, que também é acompanhado do risco dessa atividade. Entre as principais dificuldades enfrentadas tem-se a possibilidade de insucesso, necessidade de conhecimentos específicos, carência de ferramentas estruturadas de gestão e também uma série de barreiras impostas às pequenas empresas, tais como a falta de crédito junto a instituições financeiras ou investidores diretos $[1 ; 26 ; 38]$.

Somado ao rol de dificuldades expostos, nota-se em destaque a necessidade da existência de mensurações de desempenho do nível em que as diversas atividades são conduzidas pela gestão $[12 ; 14]$. Deste modo, uma das possibilidades que podem ser exploradas está contemplada neste manuscrito, que trata da verificação comparativa de fornecedores, uma vez que esta pode ser considerada uma etapa crítica devido às múltiplas perspectivas envolvidas e o reflexo direto desta escolha sobre a competitividade empresarial.

\subsection{LEVANTAMENTO DOS PVFs}

A partir das concepções metodológicas apresentadas e das considerações bibliográficas encontradas nas obras dos autores descritos naquela seção, foi possível viabilizar o levantamento dos quatro PVFs considerados como mais relevantes para a mensuração proposta.

O primeiro PVF, denominado por Custo de aquisição $(\mathrm{C} 11)$, pode ser considerado como um dos mais predominantes na avaliação de desempenho de fornecedores, de modo que muitas vezes é utilizado como único fator de mensuração, devido à concepção de que o que qualifica o serviço da empresa é quanto ela cobra para fazê-lo, mesmo com o custo baixo pelo serviço, o seu desempenho pode refletir o seu custo $[7 ; 27 ; 36]$.

Para avaliar o nível de desempenho de um fornecedor, é necessário que haja um PVF que trate exclusivamente da questão relacionada a Qualidade (C12), de modo que um dos grandes entraves encontrados no ramo empresarial está relacionado a avaliação do produto, que está diretamente vinculada ao cliente final $[11 ; 18 ; 31]$.

Ao selecionar um fornecedor um dos fatores de grande importância é a Confiabilidade (C13), pois é desejável que se escolha um fornecedor confiável, de boa reputação e financeiramente estável $[9 ; 13 ; 25]$. Para avaliar seu desempenho por meio da confiabilidade dos seus serviços, é necessário conhecer a maneira com que esta disponibiliza os seus recursos em prol do cumprimento dos compromissos firmados com os seus clientes, de modo que o aumento na confiança nesta reduz gastos com verificações de qualidade dos produtos e se as operações individuais da cadeia de suprimentos não entregarem pontualmente como prometido [6].

O último PVF está relacionado ao grau de Flexibilidade (C14) que o fornecedor possui para lidar com as mudanças e distúrbios na cadeia de suprimentos, e se ele pode se adaptar e responder de maneira adequada às flutuações de demanda da empresa contratante 
$[3 ; 18]$.

\subsection{CONSTRUÇÃO DOS PVES}

Após a determinação dos quatro pontos considerados como primordiais para a avaliação e, respectiva seleção de fornecedores de base teconógica, houve a estratificação destes em um segundo nível, a fim de contemplar de maneira mais específica o rol de fatores que influencia o contexto de decisão, onde foram identificados ao total dezessete elementos, hierarquizados segundo mostra a Tabela 1.

Tabela 1 - Estrutura hierárquica contemplando os PVFs selecionados e sua respectiva derivação em PVEs.

\begin{tabular}{|c|c|c|c|}
\hline PVF & PVE & Denominação & Definição \\
\hline $\mathrm{C} 11$ & C21 & Custo do produto & $\begin{array}{l}\text { Avalia o curso do produto em relação aos } \\
\text { concorrentes }\end{array}$ \\
\hline $\mathrm{C} 11$ & $\mathrm{C} 22$ & Frete & Avalia o custo da entrega do produto \\
\hline $\mathrm{C} 11$ & $\mathrm{C} 23$ & Custo do seguro da carga & $\begin{array}{l}\text { Avalia se a empresa possui garantia aos } \\
\text { produtos, e seu valor, e se caso este } \\
\text { apresentar defeitos, é oferecido o re-trabalho } \\
\text { da peça ou um produto novo }\end{array}$ \\
\hline $\mathrm{C} 12$ & $\mathrm{C} 24$ & Qualidade aparente na entrega & $\begin{array}{l}\text { Se o produto chega com boa aparência, sem } \\
\text { defeitos aparentes devido ao carregamento } \\
\text { ou sobreposição de caixas }\end{array}$ \\
\hline $\mathrm{C} 12$ & $\mathrm{C} 25$ & Prestação do serviço & $\begin{array}{l}\text { Verificar se a empresa cumpre o serviço da } \\
\text { maneira como compromete-se com os } \\
\text { clientes }\end{array}$ \\
\hline $\mathrm{C} 12$ & $\mathrm{C} 26$ & Desempenho do produto & $\begin{array}{l}\text { Se o produto cumpre suas funções como } \\
\text { esperado }\end{array}$ \\
\hline $\mathrm{C} 12$ & $\mathrm{C} 27$ & $\begin{array}{l}\text { Conformidade com o pré- } \\
\text { estabelecido }\end{array}$ & $\begin{array}{l}\text { Produto adequado aos padrões pré- } \\
\text { estabelecidos }\end{array}$ \\
\hline $\mathrm{C} 12$ & $\mathrm{C} 28$ & $\begin{array}{l}\text { Certificações da qualidade (ex.: } \\
\text { ISO } 9001, \ldots)\end{array}$ & $\begin{array}{l}\text { A influencia de certificações de qualidade no } \\
\text { produto }\end{array}$ \\
\hline $\mathrm{C} 12$ & $\mathrm{C} 29$ & $\begin{array}{l}\text { Histórico de performance } \\
\text { (compras anteriores) }\end{array}$ & $\begin{array}{l}\text { Avaliação do desempenho das compras } \\
\text { realizadas anteriormente }\end{array}$ \\
\hline $\mathrm{C} 13$ & $\mathrm{C} 210$ & Garantias contra extravio & $\begin{array}{l}\text { Se a empresa possuí garantias para produtos } \\
\text { extraviados }\end{array}$ \\
\hline $\mathrm{C} 13$ & $\mathrm{C} 211$ & $\begin{array}{l}\text { Atendimento a totalidade do } \\
\text { pedido }\end{array}$ & $\begin{array}{l}\text { Se o fornecedor entrega os itens solicitados } \\
\text { na compra com plenitude }\end{array}$ \\
\hline $\mathrm{C} 13$ & $\mathrm{C} 212$ & Atendimento aos prazos de entrega & $\begin{array}{l}\text { Existe a ocorrência do cumprimento dos } \\
\text { prazos estabelecidos no contrato }\end{array}$ \\
\hline $\mathrm{C} 14$ & $\mathrm{C} 213$ & $\begin{array}{l}\text { Atendimento a mudanças no } \\
\text { pedido original }\end{array}$ & $\begin{array}{l}\text { Avalia se a empresa é capaz de realizar } \\
\text { mudanças em pedidos feitos após o pedido } \\
\text { de compra original ter sido realizado }\end{array}$ \\
\hline $\mathrm{C} 14$ & $\mathrm{C} 214$ & $\begin{array}{l}\text { Capacidade } \\
\text { emergencial }\end{array}$ & $\begin{array}{l}\text { Capacidade e estrutura para lidar com } \\
\text { possíveis erros/defeitos na entrega do } \\
\text { produto }\end{array}$ \\
\hline $\mathrm{C} 14$ & $\mathrm{C} 215$ & Período necessário para entrega & Tempo necessário para entregar os materiais \\
\hline $\mathrm{C} 14$ & $\mathrm{C} 216$ & $\begin{array}{l}\text { Informações disponibilizadas } \\
\text { entrega }\end{array}$ & $\begin{array}{l}\text { Se houve rastreamento da entrega e o } \\
\text { produto cumpriu as etapas do transporte } \\
\text { corretamente }\end{array}$ \\
\hline $\mathrm{C} 14$ & $\mathrm{C} 217$ & Agilidade no atendimento & $\begin{array}{l}\text { Agilidade na resposta da empresa ao contato } \\
\text { do cliente }\end{array}$ \\
\hline
\end{tabular}

Conforme descrito na seção metodológica, para a construção da estrutura utilizou-se do método de estratificação de informações denominado por FAST, o que permitiu a obtenção de três PVEs ligados a C11 (Custo), seis ao C12 (Qualidade), três ao C13 (Confiabilidade), e 
cinco ao C14 (Flexibilidade).

\subsection{CONSTRUÇÃo MATEMÁTICA do SMD}

A construção matemática do SMD iniciou logo após a conversão dos dados coletados no "Diagnóstico 1" para as taxas de substituição dos PVEs $\left(c_{\alpha} 2 a\right)$ mensuradas com base na escala $\alpha$, Equação (1), pois a AHP está balizada a partir de duas escalas $(\varepsilon)$, a de Avaliação Fundamental desenvolvida por [32] $\varepsilon=1$, para a AHP clássica, que compreende valores entre $(1 ; 9)$, e a Natural de Lootsma [23] $\varepsilon=2$, para uma faixa contida entre $(-8 ; 8)$, utilizada para as MAHP, as quais geram as novas parametrizações para a base de dados dos fatores, descritos como $c^{\prime}{ }_{\varepsilon} 2 a$,

$$
c^{\prime}{ }_{\varepsilon} 2 a=\left\{\begin{array}{c}
\varepsilon=1 \rightarrow c_{\alpha} 2 a \times \frac{c_{1} \max }{c_{\alpha} \max } ; \forall c_{\alpha} 2 a>1 \\
\varepsilon=2 \rightarrow\left[c_{\alpha} 2 a \times c_{2} \max \right]-\left(2 \times c_{2} \max -\left|c_{2} \min \right|\right)
\end{array}\right.
$$

onde $c_{1} \max , c_{\alpha} \max$ e $c_{2} \max$ são relativos aos máximos possíveis de ser atingidos. $\mathrm{O}$ julgamento da hierarquia para a obtenção da taxa de substituição global $\left(g C_{\varepsilon} 2 a\right)$ dos PVEs ocorre, para cada técnica, por meio da construção das matrizes $\left(A_{\varepsilon} 2 a a^{\prime}\right)$ a partir da comparação paritária dos elementos localizados em um mesmo nível na estrutura que possuem relação direta, conforme mostra a Equação (2), com base nas definições propostas por [23] e [34],

$$
\mathrm{A}_{\varepsilon} 2 \mathrm{aa}^{\prime}=\left\{\begin{array}{c}
\sum_{\mathrm{a}^{\prime}=1}^{17} \frac{\mathrm{c}_{\varepsilon} 2 \mathrm{a}}{\mathrm{c}_{\varepsilon} 2 \mathrm{a} \prime} \rightarrow \varepsilon=1 \\
\sum_{\mathrm{a}^{\prime}=1}^{17} 2,7183^{\mathrm{k} \times\left(\mathrm{c}_{\varepsilon} 2 \mathrm{a}-\mathrm{c}_{\varepsilon} 2 \mathrm{a}^{\prime}\right)} \rightarrow \varepsilon=2
\end{array}\right.
$$

de modo que $k$ é a constante de proporcionalidade dos métodos multiplicativos. A seguir, em conjunto com os resultados das comparações paritárias, a Equação (3) mostra a representação da taxa de substituição de cada técnica $\left(g C_{\varepsilon} 2 a\right)$ e, por fim, de modo global $(g C 2 a)$ através da Equação (4).

$$
\begin{gathered}
\mathrm{gC}_{\varepsilon} 2 \mathrm{a}=\frac{\mathrm{A}_{\varepsilon} 2 \mathrm{aa}^{\prime}}{\sum_{\mathrm{a}=1}^{17} \sum_{\mathrm{a}^{\prime}=1}^{17} \mathrm{~A}_{\varepsilon} 2 \mathrm{aa}^{\prime}} \\
\mathrm{gC} 2 \mathrm{a}=\frac{\sum_{\varepsilon=1}^{2} \mathrm{gC}_{\varepsilon} \mathrm{ta}}{2}
\end{gathered}
$$

Como consequência da estrutura cujo perfil arbóreo hierárquico do caso, tem-se que o ranqueamento das taxas de substituição dos PVFs ( $g C 1 a)$, Equação (5), pode ser constatado a partir dos resultados estabelecidos para cada um dos PVEs em $g C 2 a$, desde que estes, necessariamente, tenham sido concebidos por meio da derivação direta e natural em relação aos seus respectivos $\mathrm{C} 1 \mathrm{a}(C 2 t \subset C 1 t)$.

$$
\mathrm{gC} 1 \mathrm{t}=\frac{\sum_{\mathrm{t}=1}^{\mathrm{n}} \mathrm{gC} 1 \mathrm{t}}{\mathrm{n}}
$$

A seguir, por meio da avaliação realizada no "Diagnóstico 2" pelos gestores das empresas (e) envolvidas, é possível incorporar os valores relativos a avaliação do desempenho dos f fornecedores em relação a cada um dos PVEs estabelecidos $\left(p f_{C 2 a}\right)$, em relação as taxas globais dos fatores $\left(g C_{\varepsilon} t a\right)$, conforme as diretrizes propostas pela Equação (6), geram o índice de proporcionalidade sistêmico $p_{e f} C_{\varepsilon} 2 a$ proposto na Equação (6),

$$
\mathrm{p}_{\mathrm{ef}} \mathrm{C}_{\varepsilon} 2 \mathrm{a}=\mathrm{gC}_{\varepsilon} 2 \mathrm{a} \times \mathrm{pf}_{\mathrm{C} 2 \mathrm{a}} \rightarrow \mathrm{t}=2
$$


de maneira que o valor relativo percentual $\left(r_{e f} C_{\varepsilon} 2 a\right)$ pode ser observado através da relação entre as pontuações absolutas calculadas em $p_{e f} C_{\varepsilon} 2 a$, a partir dos pressupostos descritos na Equação (7). Por consequência, a Equação (8) mostra os resultados sob a ótica global, por meio da reunião dos valores de $r_{e f} C_{\varepsilon} 2 a$ estabelecidos individualmente em ambos métodos multicritério adotados.

$$
\begin{gathered}
\mathrm{r}_{\mathrm{ef}} \mathrm{C}_{\varepsilon} 2 \mathrm{a}=\frac{\mathrm{p}_{\mathrm{ef}} \mathrm{C}_{\varepsilon} 2 \mathrm{a}}{\sum_{\mathrm{f}=1}^{\mathrm{n}} \mathrm{p}_{\mathrm{ef}} \mathrm{C}_{\varepsilon} 2 \mathrm{a}} \times 100 \\
\mathrm{r}_{\mathrm{ef}} \mathrm{C} 2 \mathrm{a}=\frac{\mathrm{r}_{\mathrm{ef}} \mathrm{C}_{\varepsilon} 2 \mathrm{a}}{\sum_{\varepsilon=1}^{2} \mathrm{r}_{\mathrm{ef}} \mathrm{C}_{\varepsilon} 2 \mathrm{a}}
\end{gathered}
$$

A fim de conceber o desempenho do fornecedor em proporção aos PVEs determinados pela estrutura hierárquica, tem-se que o índice $E_{e f \varepsilon}$ é definido de acordo com a média dos valores relativos $r_{e f} C_{\varepsilon} 2 a$, segundo a Equação (9),

$$
\mathrm{E}_{\mathrm{ef} \varepsilon}=\frac{\sum_{\mathrm{a}=1}^{17} \mathrm{r}_{\mathrm{ef}} \mathrm{C}_{\varepsilon} 2 \mathrm{a}}{17}
$$

no qual, em um segundo momento, serve como base para a avaliação de desempenho das empresas adotadas, de forma a contemplar a média dos valores mensurados em todas as metodologias $\left(E_{e f}\right)$, no caso a AHP $(\varepsilon=1)$ e a MAHP $(\varepsilon=2)$, como mostra a Equação $(10)$.

$$
\mathrm{E}_{\mathrm{ef}}=\frac{\sum_{\varepsilon=1}^{2} \mathrm{E}_{\mathrm{ef} \varepsilon}}{2}
$$

Quanto a demanda de obtenção dos resultados global que relacionem os PVF ( $t=$ 1), basta parametrizar as relações de variáveis de acordo com a estrutura hierárquica elaborada até se chegar ao índice $r_{e f} C 1 a$, o que gera cuidados extras principalmente quanto a verificação da obrigatoriedade de vínculo dos fatores ao seu nível diretamente superior, de acordo com a relação $C_{\varepsilon} t a \subset C_{\varepsilon}(t-1)$.

Para fins de constatação do grau de confiabilidade da modelagem, [33] e [35] dissertam a respeito da necessidade de se realizar o cálculo da consistência lógica dos julgamentos realizados em cada um dos níveis da estrutura, partindo do pressuposto que os avaliadores da situação podem ser, no momento da tomada da decisão, inconsistentes com relação à definição dos pesos para os fatores.

Para tanto, a AHP incorpora essas situações possibilitando ao usuário a verificação do nível de contradição em cada etapa, assegurando o seu grau de confiabilidade quanto mais próximo for o valor da igualdade entre $\mathrm{n}$ e $\lambda_{\max }\left(\lambda_{\max }=n\right)$, podendo-se considerar que as diferenças dessas reciprocidades sejam consideradas nulas ou muito pequenas, onde a medida dessa relação é apresentada através do índice de consistência $\left(I C_{A i}\right)$ mostrado pela Equação (11) com base em [34],

$$
\mathrm{IC}_{\mathrm{Ai}}=\frac{\lambda_{\max }-\mathrm{n}}{\mathrm{n}-1}
$$

sendo $A i=\left\{A_{1} a t t^{\prime} ; A_{3} a t t^{\prime} ; A C 2 t_{1} I d d^{\prime}\right\}$ referente as matrizes de julgamentos que tem por diretriz a utilização da AHP. Por consequência o cálculo da razão de consistência $\left(C M_{A i}\right)$ através da Equação (12), permitindo finalmente definir a confiabilidade de cada uma das matrizes de julgamentos da modelagem, 


$$
\mathrm{CM}_{\mathrm{Ai}}=\left(\frac{\mathrm{IC}}{\mathrm{IR}}\right)_{\mathrm{Ai}}
$$

onde $I R_{A i}$ é definido como o índice de consistência randômico, calculado a partir de testes de diversas matrizes quadradas de ordem $\mathrm{n}$ padronizada a partir do Laboratório Oak Ridge, localizado nos Estados Unidos [15]. Em caso de $C M_{A i}$ exceder o limite tolerável para o modelo $(\geq 0,1)$, o tomador de decisão deve realizar a revisão das comparações definidas atribuindo novos valores para os subcritérios envolvidos no julgamento proposto [35].

\section{APLICAÇÃO DA MODELAGEM}

De modo a contemplar a discussão prática acerca da modelagem desenvolvida, foi proposta a sua aplicação ao contexto da Incubadora Tecnológica de Santa Maria (ITSM), localizada e fomentada pela Universidade Federal de Santa Maria (UFSM). Para tanto, o primeiro passo tomado foi a aplicação do "Diagnóstico 1" ao gerente responsável pela condução das ações na incubadora, no qual remeteu a sua opinião acerca do nível de relevância dos PVFs e PVEs elencados ao decorrer da estrutura hierárquica do SMD.

Após a realização dos questionamentos houve a compilação e, respectivamente, o julgamento da situação global de cada um dos fatores, a partir dos valores disponibilizados pelo entrevistado, onde chegou-se ao estabelecimento das taxas de substituição mostradas na Figura 2.

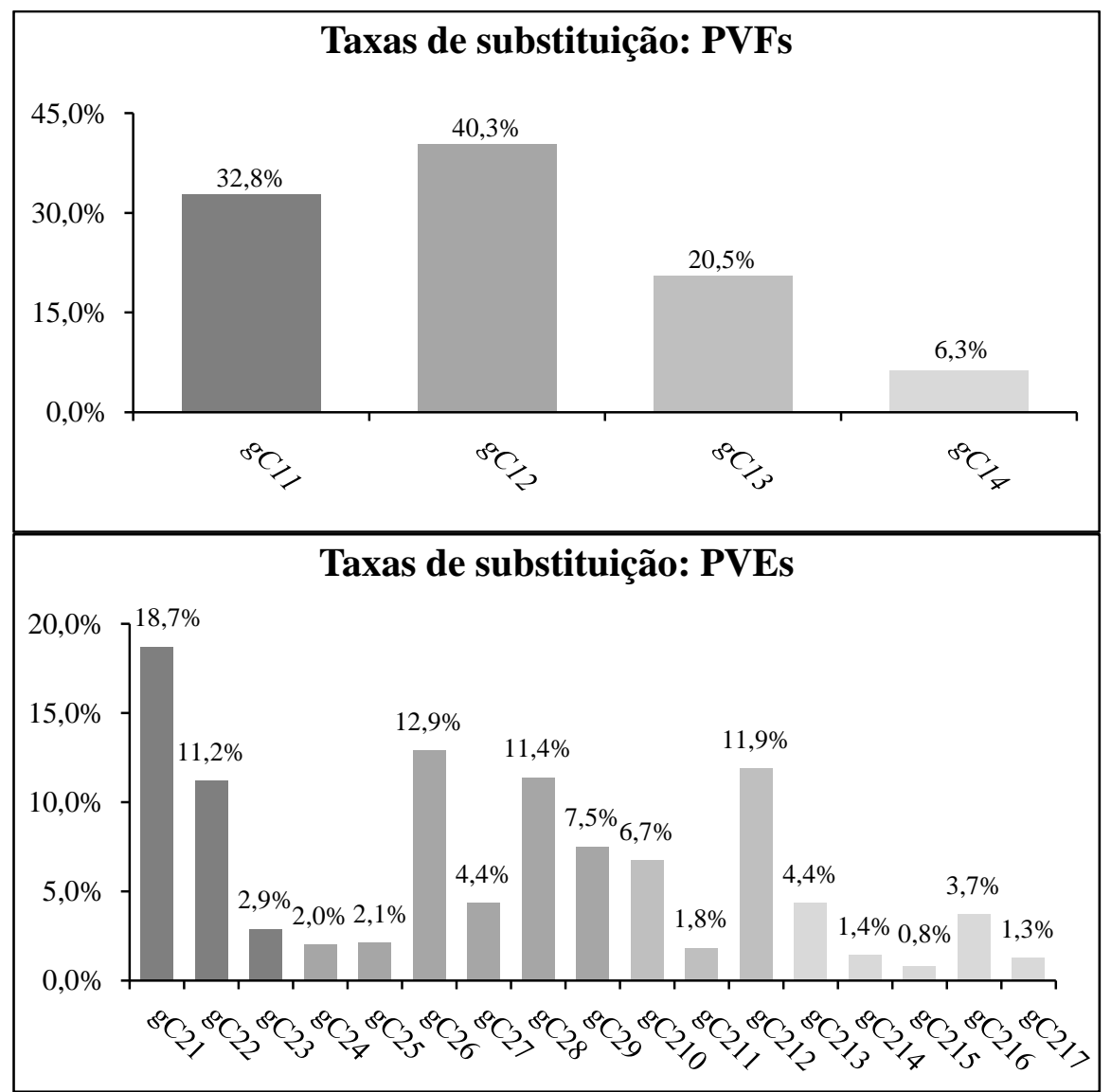

Figura 2 - Taxas de substituição dos PVFs e PVEs elencados no SMD $(k=0,2)$.

Para $k=0,2$ que minimiza a função $e k$ em 0,371 pontos, notou-se que para os PFVs a maior taxa ficou estabelecida para o critério C12 (Qualidade), com mais da metade da pontuação possível de ser atingida ( $g C 12=40,3 \%$ ), seguido do C11 (Custo de Aquisição), que absorveu aproximadamente um terço da proporção total $(g C 11=32,8 \%)$. Se 
considerarmos o somatório de ambos, é possível perceber que $73,1 \%$ da relativização está concentrada nestes, de modo que tal indicador denota o nível de preocupação que os fornecedores devem ter com o impacto em que os seus produtos estão causando, positivamente ou negativamente, para com as empresas contratantes em relação aos quesitos acima descritos. Ainda, C13 (Confiabilidade) ficou com o terceiro maior conceito ( $g C 13=$ 20,5\%) e, por último, C14 (Flexibilidade) foi o considerado como de menor influência no desempenho $(g C 14=6,3 \%)$.

Para os dezessete PVEs julgados, cinco foram capazes de superar a marca de $10 \%$, C21 (Custo do produto: $g C 21=18,7 \%$ ), C26 (Desempenho do produto: $g C 26=12,9 \%$ ), C212 (Atendimento aos prazos de entrega: $g C 26=11,9 \%$ ), C28 (Certificações da qualidade: $g C 28=11,4 \%$ ) e C22 (Frete: $g C 22=11,2 \%$ ), dos quais por consequência se tornaram os mais relevantes a serem considerados para a tomada de decisão em questão.

Quanto aos cinco PVEs menos relevantes, pode-se destacar que os três piores colocados estão contidos a estrutura de C14, C215 (Período necessário para entrega: $g C 215=0,8 \%$ ), C217 (Agilidade no atendimento: $g C 217=1,3 \%$ ), C214 (Capacidade de atender a demandas emergenciais: $g C 214=1,4 \%$ ), o que ressalta o papel secundário deste PVF ao contexto. Ainda, estão contidos nessa categoria os PVEs C211 (Atendimento a totalidade do pedido: $g C 211=1,8 \%$ ), relacionado a Confiabilidade, e C24 (Qualidade aparente na entrega: $g C 24=2 \%$ ).

Em relação a consistência lógica das cinco matrizes de julgamentos, tem-se que a medida de $\mathrm{CM}_{\mathrm{Ai}}$ não superou a marca limite de $10 \%$ supracitada, o que indica que a opinião dos decisores, nos dois diagnósticos, foi coerente ao decorrer do processo de mensuração.

Em relação a mensuração de desempenho dos fornecedores, conforme mostrado na seção metodológica, houve a seleção das três empresas instaladas que possuem condições técnicas de avaliar o desempenho dos seus fornecedores, principalmente quanto dos seus níveis consolidados de maturação no processo de incubação. Quanto as características de cada negócio, a empresa $1(e=1)$ atua com a criação de produtos e projetos na área de eletrônica embarcada, a empresa $2(e=2)$ é voltada para a pecuária de precisão, e a empresa $3(e=3)$ tem como foco conceber e criar sistemas inteligentes para a produção de água potável reutilizável.

Ao término da verificação preliminar acerca do funcionamento básico dos empreendimentos, foi possível viabilizar, em conjunto com os decisores, a aplicação do “Diagnóstico 2", partindo-se do pressuposto que estes deveriam avaliar a situação dos fornecedores, denominados por $\mathrm{A}(f=1)$ e $\mathrm{B}(f=2)$, de modo que os resultados obtidos da mensuração são mostrados na Tabela 2.

Tabela 2 - Resultados da mensuração de desempenho dos fornecedores elencados as três empresas.

\begin{tabular}{ccccccccccc}
\hline \multirow{2}{*}{ Empresa } & \multirow{2}{*}{ Total } & \multicolumn{3}{c}{ Custo (C11) } & \multicolumn{2}{l}{$\begin{array}{l}\text { Qualidade } \\
\text { (C12) }\end{array}$} & \multicolumn{2}{l}{$\begin{array}{l}\text { Confiabilidade } \\
\text { (C13) }\end{array}$} & \multicolumn{2}{l}{$\begin{array}{l}\text { Flexibilidade } \\
\text { (C14) }\end{array}$} \\
\cline { 2 - 10 } & $f=1$ & $f=2$ & $f=1$ & $f=2$ & $f=1$ & $f=2$ & $f=1$ & $f=2$ & $f=1$ & $f=2$ \\
\hline$e=1$ & $47,1 \%$ & $52,9 \%$ & $44,6 \%$ & $55,4 \%$ & $47,3 \%$ & $52,7 \%$ & $52,1 \%$ & $47,9 \%$ & $39,0 \%$ & $61,0 \%$ \\
$e=2$ & $57,6 \%$ & $42,4 \%$ & $61,2 \%$ & $38,8 \%$ & $55,8 \%$ & $44,2 \%$ & $52,0 \%$ & $48,0 \%$ & $63,3 \%$ & $36,7 \%$ \\
$e=3$ & $53,0 \%$ & $47,0 \%$ & $42,7 \%$ & $57,3 \%$ & $57,2 \%$ & $42,8 \%$ & $68,5 \%$ & $31,5 \%$ & $86,2 \%$ & $13,8 \%$ \\
\hline
\end{tabular}

Para $e=1$ a pontuação final dos fornecedores ficou estabelecida por $E_{11}=47,1 \% \mathrm{e}$ $E_{12}=52,9 \%$, de maneira que a maior discrepância verificada está identificada para a Flexibilidade, onde B $\left(r_{12} C 14=61 \%\right)$ apresentou um resultado melhor que A $\left(r_{11} C 14=\right.$ $39 \%)$, devido basicamente a boa situação de fornecimento encontrada em C214 ( $r_{11} C 214=$ $36,4 \%$ e $\left.r_{12} C 214=63,6 \%\right)$ e C215 $\left(r_{11} C 215=42,9 \%\right.$ e $\left.r_{12} C 215=57,1 \%\right)$. Ainda, constatou-se que houve uma inversão dos valores em relação ao padrão estabelecido na Confiabilidade, de modo que A atingiu uma aceitação de 52,1\% em relação a $\mathrm{B}$, proveniente 
do resultado estabelecido em C212 $\left(r_{11} C 212=52,9 \%\right.$ e $\left.r_{12} C 212=63,6 \%\right)$, corroborado ao fato que este possui a terceira maior taxa de substituição global $(g C 212=11,9 \%)$ em relação aos demais PVEs.

$\mathrm{Na}$ avaliação de $e=2$ encontrou-se uma diferença considerável entre os desempenhos medidos, onde o fornecedor A se sobressaiu em todos os PVFs, principalmente nas melhores condições apresentadas no Custo $\left(r_{21} C 11=61,2 \%\right)$ e Flexibilidade $\left(r_{21} C 14=\right.$ $63,3 \%$ ), sendo que a ultima apresentou a maior disparidade da avaliação, padrão que se perpetuou devido ao perfil de superioridade médio de 40,05\% em relação aos PVEs elencados em C14. O único resultado averso foi o estabelecido em C212 $\left(r_{22} C 212=58,3 \%\right)$, o que, apesar de ser um ponto favorável, acaba sendo irrelevante ao contexto.

Em $e=3$, os fornecedores apresentaram desempenho próximo, onde o fornecedor A se destacou comparado a B em $6 \%$, devido principalmente a boa avaliação obtida em três dos quatro PVFs: Qualidade $\left(r_{31} C 12=57,2 \%\right)$, Confiabilidade $\left(r_{31} C 13=68,5 \%\right) \quad$ e Flexibilidade $\left(r_{31} C 14=82,6 \%\right)$, cabendo um destaque especial a este último, devido a elevada diferença média de competitividade existente entre ambos, $71,05 \%$, se considerados os resultados estabelecidos em seus PVEs. Ainda, constatou-se que apenas em outras quatro oportunidades (C26, C27, C212 e C213) os resultados de A foram superiores a B, e em três momentos houve igualdade entre as relações (C28, C29 e C210).

\section{CONCLUSÕES}

Após a compilação dos resultados, notou-se que as maiores diferenças na avaliação do desempenho dos fornecedores ficaram concentradas nos PVFs Flexibilidade e Custo, seguidos por Confiabilidade e, por último, a Qualidade, que foi o mais semelhante entre os avaliados. Já nos PVEs a maior diferença deu-se nos indicadores de Confiabilidade, onde as principais disparidades foram atendimento a mudanças no pedido, seguido por garantias contra extravio e atendimento a mudanças. Somado a isso, os PVEs Custo do produto, Desempenho do produto e Atendimento aos prazos de entrega podem ser considerados como os mais importantes para a criação de vantagens competitivas, devido às taxas de substituição apresentadas.

Em relação ao objetivo proposto, constatou-se que o tipo de modelagem matemática aplicada é capaz de remeter resultados fidedignos a realidade do setor estudado, de maneira a retomar, independente da natureza de operação dos Fatores Críticos de Sucesso (FCS) elencados, quantitativamente a situação dos fornecedores submetidos a verificação, diferenciando-se portanto das tradicionais formas de verificação existentes.

Como limitações encontradas ao decorrer do desenvolvimento, tem-se a dificuldade dos gestores em determinar, preliminarmente a aplicação do modelo, quais devem ser os fornecedores que podem participar da mensuração, pois a aplicação foi proposta contemplando apenas a escolha entre dois fornecedores. Em relação às expectativas futuras, espera-se que novos testes com a ferramenta, incluindo a análise de sensibilidade, possibilite novas formas de se visualizar a maneira com que vem se comportando o contexto dos fornecedores para corporações de base tecnológica, a fim de contribuir para a obtenção da qualidade do produto final.

\section{REFERÊNCIAS BIBLIOGRÁFICAS}

[1] ANPROTEC. Estudo, Análise e Proposições sobre as Incubadoras de Empresas no Brasil - relatório técnico. Ministério da Ciência, Tecnologia e Inovação: Brasília, 2012.

[2] BERGEK, A.; NORRMAN, C. Incubator best practice: A framework. Technovation, v. 28, n. 1-2, p. 20-28, 2008.

[3] BLANCHARD, D. Supply Chain Management Best Practices. Hoboken: Wiley, 2010. 
[4] BOWERSOX, D.; CLOSS, D.; COOPER, M.B. Supply Chain Logistics Management. Columbus: McGraw-Hill/Irwin, 2012.

[5] BYTHEWAY, C.W. Fast Creativity \& Innovation: Rapidly Improving Processes, Product Development and Solving Complex Problems. Plantation: J. Ross Publishing, 2007.

[6] CARTER, J.R.; MALTZ, A.; MALTZ, E.; GOH, M.; YAN, T.T. Impact of culture on supplier selection decision making. International Journal Of Logistics Management, v. 21, n. 3, p.353-374, 2010.

[7] CHO, G.; SOH, S. Optimal decision-making for supplier-buyer's maximum profit in a two echelon supply chain. African Journal Of Business Management, v.4, n. 5, p.687694, 2010.

[8] CHOPRA, S.; MEINDL, P. Supply Chain Management. Lebanon: Prentice Hall, 2012.

[9] COUSINS, P.D.; LAWSON, B.; SQUIRE, B. Performance measurement in strategic buyer-supplier relationships: The mediating role of socialization. International Journal Of Operations \& Production Management, v. 28, n. 3, p.238-258, 2008.

[10] DAVIS, M.D.; AQUILANO, N.J.; CHASE, R.B. Fundamentos da Administração da Produção. Porto alegre: Bookman, 2003.

[11] DURSUN, M.; KARSAK, E.E. Supplier Selection Using an Integrated Decision Making Approach Based on QFD and 2-Tuple Fuzzy Representation. Lecture Notes in Engineering and Computer Science, v. 2201, n. 1, p.1309, 2012.

[12] ESTAMPE, D.; LAMOURI, S.; PARIS, J.L.; BRAHIM-DJELLOUL, S. A framework for analysing supply chain performance evaluation models. International Journal of Production Economics, v. 142, p. 247-258, 2013.

[13] FENG, B. ; FAN, Z.-P. ; LI, Y. A decision method for supplier selection in multiservice outsourcing. International Journal of Production Economics, v. 132, n. 2, p.240$25,2011$.

[14] FERREIRA, M. P.; ABREU, A. F.; ABREU, P. F.; TRZECIAK, D. S.; APOLINÁRIO, L. G.; CUNHA, A. d'Ávila. Gestão por indicadores de desempenho: resultados na incubadora empresarial tecnológica. Produção, v.18, n.2, 2008.

[15] GOMES, C.F.; GOMES, L.F.A.M. Tomada de decisão gerencial: Enfoque Multicritério. 4 ed. São Paulo: Atlas, 2012.

[16] HUBBARD, G.; RICE, J.; BEAMISH, P. Strategic Management: Thinking, Analysis and Action. Pearson Education, 2011.

[17] IRELAND, R.D.; HOSKISSON, R.E.; HITT, M.A. Strategic Management: Concepts and Cases: Competitiveness and Globalization. Boston: South-Western College Publishing, 2014.

[18] JACOBS, F.R.; CHASE, R. Operations and Supply Chain Management. Columbus: McGraw-Hill/Irwin, 2013.

[19] JUGEND, D.; SILVA, S.L. Práticas de gestão que influenciam o sucesso de novos produtos em empresas de base tecnológica. Produção, v. 20, n. 3, p. 335-346, 2010.

[20] KAPLAN, R.S.; NORTON, D.P. The Execution Premium: Linking Strategy to Operations for Competitive Advantage. Boston: Harvard Business Review Press, 2008.

[21] LAUGENI, F.P.; MARTINS, P.G. Administração da Produção. São Paulo: Saraiva, 2006. 
[22] LI, L.; QIAN, G.; QIAN, Z. The performance of small and medium-sized technologybased enterprises: do product diversity and international diversity matter?

[23] LOOTSMA, F.A. Scale sensivity in the multiplicative AHP and Smart. Journal of Multi-criteria Decision Analysis, v. 2, p. 87 - 110, 1993.

[24] MUKHOPADHYAYA, A.K. Function Analysis System Technique. 1 ed. : Andhra Pradesh: I K International Publishing House, 2012.

[25] MYERSON, P. Lean Supply Chain and Logistics Management. Columbus: McGrawHill, 2012.

[26] NBIA. National Business Incubation Association. Disponivel em: <http://www.nbia.org/ about_nbia/>. Acessado em: 17 fev. 2014.

[27] NIKOLAEVNA, L.O. Organizing-economical decision in supplier selection. Vestnik of Astrakhan State Technical University. Series: Economics, v. 2, p. 54, 2012.

[28] PARMENTER, D. Key Performance Indicators (KPI): Developing, Implementing, and Using Winning KPIs. 2 ed. Wiley: Hoboken, 2010.

[29] PETROU, A., LIARGOVAS, P., DASKALOPOULOU, I. Entrepreneurship incubators and economic growth. New York: Nova Science, 2010.

[30] Phan, P. H., Siegel, D. S. and Wright, M. (2005). Science parks and incubators: observations, synthesis and future research. Journal of Business Venturing Vol: 20, No: 2: $165-182$.

[31] PORTER, M.E. (2009). On Competition, Updated and Expanded Edition, Watertown; Harvard Business Press.

[32] SAATY, T.L. The analytic hierarchy process. New York: McGraw-Hill, 1980.

[33] SAATY, T.L. Decision-making with the AHP: Why is the principal eigenvector necessary. European Journal of Operational Research, v. 145, p. 85-91, 2003.

[34] SAATY, T.L. Decision making with the Analytic Hierarchy Process. International. Journal of Services Sciences, v. 1, n. 1, p. 83 - 98, 2008.

[35] SAATY, T.L.; CHANG, J.S. An innovative orders-of-magnitude approach to AHPbased mutli-criteria decision making: Prioritizing divergent intangible humane acts. European Journal of Operational Research, 214, 703 - 715, 2011.

[36] SLACK, N.; CHAMBERS, R.; JOHNSTON, R.; BETTS, A. Operation and process management: principles and practice for strategic impact. Prentice Hall, Lebanon, 2008.

[37] TOLEDO, J.C. Silva, S.L., Mendes, G.H.S., Jugend, D. Fatores críticos de sucesso no gerenciamento de projetos de desenvolvimento de produto em empresas de base tecnológica de pequeno e médio porte. Gestão \& Produção, v. 15, n. 1, p. 117-134,2008.

[38] VOHORA, A.; WRIGHT, M.; LOCKETT, A. Critical junctures in the development of university high-tech spinout companies. Research Policy, v. 33, n. 1, p. 147-175, 2004. 